\title{
DESIGN AND IMPLEMENTATION OF LABVIEW BASED SCADA FOR TEXTILE MILLS
}

\author{
Nirmal Bharatia $^{1}$, N. D. Thakur ${ }^{2}$ \\ ${ }^{1}$ Electronics Dept., MPSTME, NMIMS, Mumbai, India \\ ${ }^{2}$ Assistant Professor Electronics and Communication Dept., MPSTME, NMIMS, Mumbai, India
}

\begin{abstract}
Quality is the watchword of any type of business. A product without quality leads to lack of customer satisfaction and ultimately to loss. This is true in case of textile industries also. In textile industries, quality maintenance is one of the major problems for fabric manufacturers. Currently in indigenous small scale textile industries manual or semi-automatic processes are being carried out. The fabric obtained from such processes lacks consistency in quality, moreover these industries have semi- skilled or unskilled labors, therefore stringent supervision is required. Normal automation solutions for such industries will be unaffordable. Therefore it is required to provide economical, reliable and robust automation solution to such industries. This paper addresses the need of factory automation within the scope of machine control for small scale textile industries. The project presents the design of LabVIEW based SCADA system for centralized control. It makes use of PLC as a field controller to operate the prototype design of Stenter Machine, widely used in textile industries. LabVIEW, which is a commonly accepted graphical user interface environment, also provides HMI front end. The PLC controller and the LabVIEW based SCADA are communicating through the RS-232 link. The control system is flexible and modular. Due to the intuitive programming of LabVIEW, this system is cost efficient and reliable solution for automation of small scale textile industries.
\end{abstract}

Keywords - SCADA; Programmable Logic Controller (PLC); LabVIEW; Stenter Machine; Printing Machine.

\section{INTRODUCTION}

The Indian small scale textile production has a major impact on the world economy through millenniums. At present fabric inspection depends on human sight, the result of inspection influenced by the physical and mental condition of inspector. Now, all the textile industries aim to produce good quality fabrics with high production rate. In the textile sector, there are huge losses due to faulty fabrics.

The faults found in the fabrics are around $80-85 \%$ of the defects in the garment industry. These faults are obtained in the fabrics due to irregular stretching and shrinking of the cloth. The manual inspection of fabric material is not economical and work is very dull. Hence, the investment in automated fabric defect detection is economical when reduction in labor cost and other benefits are considered.

In recent years, PC-based control technologies have become widely used industry practice. Benefits include faster design cycles, lower downtime using diagnostics and simulation tools, increased productivity and decreased maintenance costs. Moreover, open system designs that use standard hardware and operating system software minimize cost, permit system scalability, and ensure future performance enhancement [1].

One major development from the utility of the PC is the concept of Virtual Instrumentation, which offers several benefits to engineers and scientists who aim increased productivity, accuracy, and performance. Engineers and scientists, whose needs, applications and requirements change very quickly, need flexibility to create their own solutions. One can adopt a virtual instrument to one particular need without having to replace the entire device because of application software installed on the PC and the wide range of available plug-in hardware [2].

The power of Virtual Instruments (VI) application software, LabVIEW lies in the fact that it empowers the user to include test equipment as objects in their programs [3]. LabVIEW, as a programming language, is a powerful tool that can be used to help achieve these goals. LabVIEW (Laboratory Virtual Instrument Engineering Workbench) is a graphically-based programming language developed by National Instruments. Its graphical nature makes it ideal for test and measurement (T\&M), automation, instrument control, data acquisition, and data analysis applications. This results in significant productivity improvements over conventional programming languages. National Instruments focuses on products for T\&M, giving them a good insight into developing LabVIEW.

The main objective of this paper is to derive a brief idea about a basic level control scheme through a defined logic and a visual experience. This paper can be used at its 
higher level as it will provide the basic design guidelines for the one who wants to implement it on an industry level. The section 2 in this paper discusses concept of SCADA, section 3 provides the information about LabVIEW. The design of system is presented in section 4 , followed by the discussion of system software in section 5. Section 6 discusses about the implementation of the designed system. The results and discussion are offered in section 7 and finally conclusion in the section 8 .

\section{SCADA SYSTEM}

The supervisory control and data acquisition (SCADA) system is the backbone of modern industry. It provides centralized control and monitoring of processes with data logging.

\subsection{Need for SCADA System}

The industrial control systems (ICS), which include SCADA systems, distributed control systems (DCS), and other smaller control system configurations such as skid-mounted Programmable Logic Controllers (PLC) are often found in the industrial control sectors. ICSs are typically used in industries such as electric, water, oil and gas, transportation, chemical, pharmaceutical, pulp and paper, food and beverage, textile industry and other discrete manufacturing processes.

SCADA systems are highly distributed systems used to control geographically dispersed assets, often scattered over thousands of square kilometers, where centralized data acquisition and control are critical to system operation. They are used in distribution systems such as water distribution and wastewater collection systems, oil and gas pipelines, electrical power grids, and railway transportation systems. A SCADA control center performs centralized monitoring and control for field sites over long-distance communications networks, including monitoring alarms and processing status data. Based on information received from remote stations, automated or operator-driven supervisory commands can be pushed to remote station control devices, which are often referred to as field devices. Field devices control local operations such as opening and closing valves and breakers, collecting data from sensor systems, and monitoring the local environment for alarm conditions.

\subsection{PLC as a Field Controller}

A Programmable controller is a solid state user programmable control system with functions to control logic, sequencing, timing, arithmetic data manipulation and counting capabilities. It can be viewed as an industrial computer that has a central processor unit, memory, input output interface and a programming device. The central processing unit provides the intelligence of the controller.

PLC is special-purpose industrial computers designed for use in the control of wide variety of manufacturing machine and system. PLC also can be defined as a specialized electronic device based on one or more microprocessors that are used to control industrial machinery. The term "industrial" on the definition implies that PLCs are computer designed to operate in harsh physical and electrical noise environment present in production plants. The definition defined that PLCs are computers; hence they must be programmed using a programming language. There are five standard programming languages standardize by IEC including Ladder Diagrams (LD), Function Block Diagrams (FBD), Structured Text (ST), Instruction List (IL) and Sequential Function Charts (SFC) [4]. Here Delta DVP- 14SS2 PLC is being used. Section 5 discusses the PLC and its working in detail.

\section{LABVIEW}

LabVIEW provides the flexibility of integration of data acquisition software/hardware with the process control application software for automated test and measurement applications. By LabVIEW-aided PLC controller, the parameters are adjusted to control the motor and various other parameters [5].

The information development is complicated by the mix of hardware and software that exists in plants and the limitation of traditional systems. We can incorporate LabVIEW into existing system to add measurements and analysis to processes to gather complex data and convert it into useful information. Additionally, through the openness of LabVIEW we can connect and provide the information where it is needed, whether that is in a database [6].

The LabVIEW graphical programming paradigm simplifies the development of your distributed monitoring and control systems. Creating your application is as simple as dragging and dropping graphical functions and wiring the objects together to form a dataflow program. Built-in libraries include resources from general programming functions to powerful, applicationspecific routines [7]. Construct interactive user interfaces from hundreds of included objects, such as charts and graphs, numerical representations, and Boolean operators. LabVIEW also features robust debugging tools, including probes, breakpoints, execution highlighting, and the ability to single step through your code. In LabVIEW, you build a user interface with a set of tools and objects. The user interface is known as the front panel. You then add code using graphical representations of functions to control the front panel objects. The block diagram contains this code. In some ways, the block diagram resembles a flowchart.

\subsection{Front Panel}

The front panel is the user interface of the VI. The Fig. 1 shows an example of a front panel. You build the front panel with controls and indicators, which are the interactive input and output terminals of the VI, respectively. The Fig. 1 shows the front panel of Stenter Machine used in textile mills. The 
function of Stenter Machine is to stretch the cloth. The Fig. 1 shows the GUI of full Stenter Machine.

\subsection{Block Diagram}

After you build the front panel, you add code using graphical representations of functions to control the front panel objects. The block diagram contains this graphical source code. Front panel objects appear as terminals on the block diagram. The VI in Fig. 2 shows several primaries block diagram objectsterminals, functions, and wires. Fig. 2 is also called as a back panel, here the connections are made of the Stenter Machine. The front panel operates only after giving proper connections on back panel. If the connections are not made in back panel of VI, the functioning and working will not be reflected on the front panel.

\subsection{Advantages of LabVIEW}

- Graphical user interface.

- Drag-and-drop built in functions.

- Works on Multi platforms.

- Multiple high level development tools.

- Flexibility and scalability.

- Connectivity and instrument control.

- Simple application distribution.

- Open environment.

- Distributed development.

- Compiled languages for fast execution.

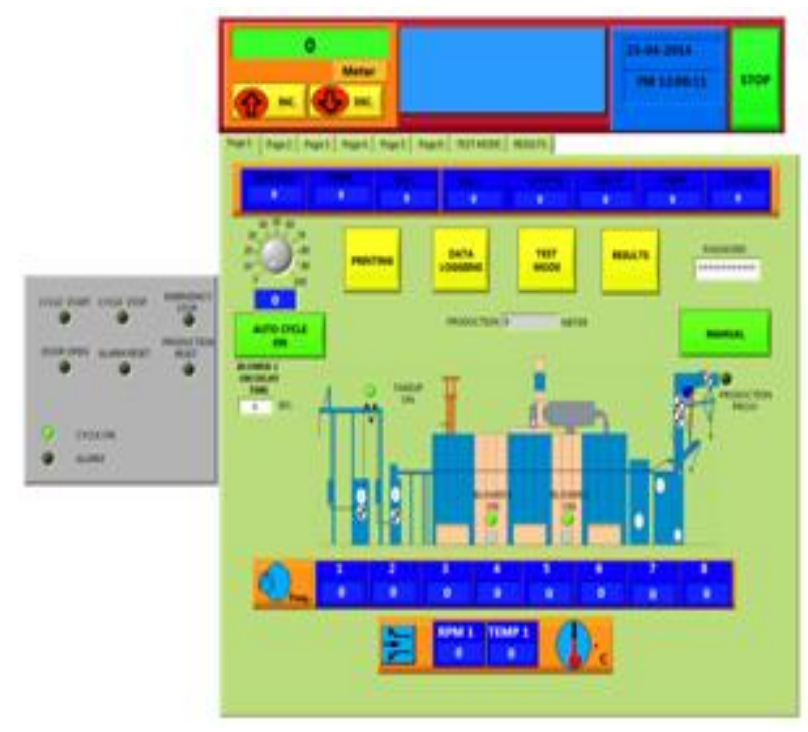

Fig 1 Front Panel in LabVIEW

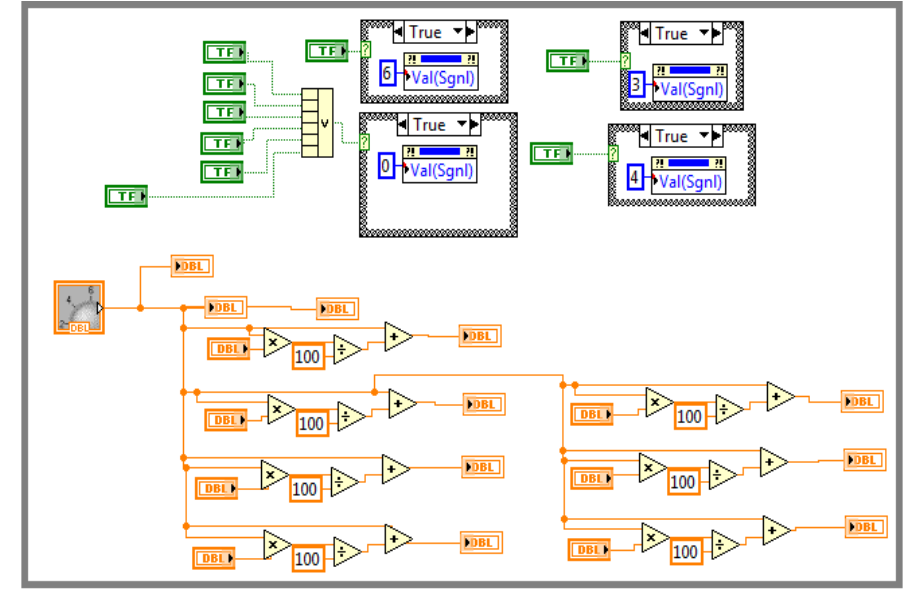

Fig 2 Block Diagram in LabVIEW

\section{DESIGN OF SYSTEM HARDWARE}

This section gives the overview of the complete system hardware. A general block diagram of the proposed system is given in Fig. 3. It gives the general overview of the system created and how it will operate.

The designed SCADA is for Stenter Machine and Printing Machine which is widely used in textile mills. The simulation created on LabVIEW is further implemented on a prototype of a Stenter Machine. The control for the implemented machine can be automatic or manual mode.

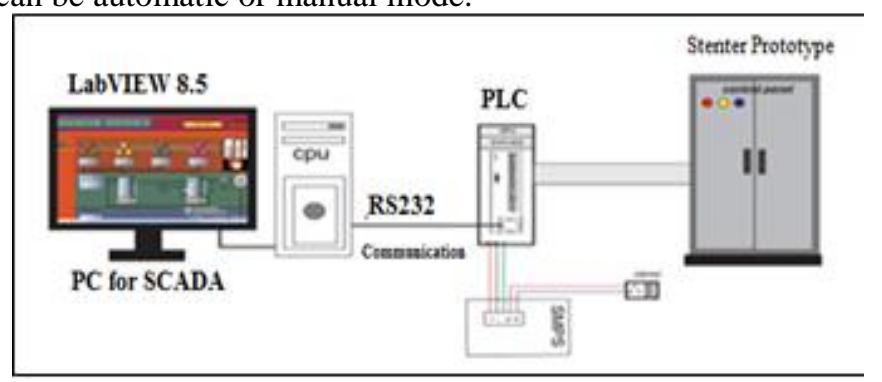

Fig 3 Proposed System Block Diagram

In automatic mode, the prototype of Stenter Machine is operated by GUI designed on LabVIEW. The machine is controlled by the computer and the data is logged and saved on an excel file created on the computer. The system is made secure by giving a password to the GUI. The machine's data logging and production page opens only after giving the password. This helps the operator to keep the output safe from others. The prototype of Stenter Machine can also be operated manually. The Fig. 4 shows the manual operator panel. If there is some fault in computer and the machine has to be operated, one can switch over to manual mode. Even in the LabVIEW a command is generated to switch to manual mode. 


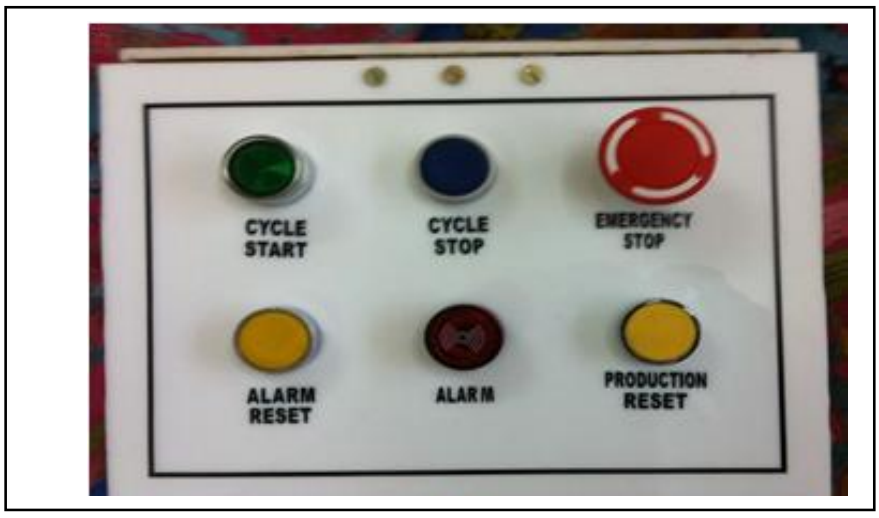

Fig 4 Manual Operation Panel

These buttons will send command to the PLC and the command from PLC will further operate the Stenter machine. First is the manual start button. This button is connected to the input pin of the PLC, which will accept the command from the user. It will start the prototype of the Stenter machine. The prototype will run as per the program in PLC. Second button is for cycle stop. Whole system can be stopped by pressing it. Similar is the function for emergency stop button. The only difference is that emergency stop is on field operation. It cannot be operated through SCADA system. But if this button is pressed, the information will be sent to the operator using SCADA. The operator will come to know when this button is pressed. The forth button represents the alarm reset. If a fault occurs, the alarm will ring (Fig. 4 fifth button), after clearing the fault the operator has to reset the alarm. This resetting of alarm is possible only if the fault is cleared. If the fault is not cleared, the alarm will keep ringing and one cannot reset it. This reset can be done on field only. The indication of alarm and alarm reset is reflected on the SCADA system.

The last button is for resetting the production. If production for a day is achieved but one does additional production, he may remove the required batch and continue for another batch. At this point of time he may not stop the machine, he can just remove the batch from the end and keep another roller where the other batch is rolled. When another batch starts he has to reset the production count. This reset can be done just by pressing the production reset button. These all buttons are connected to the PLC and further connected to the prototype of Stenter machine.

Fig. 5 shows the complete system hardware design. The prototype of Stenter Machine consists of six 5V DC motors which act as blowers of the Stenter Machine. These 5VDC motors are powered by IC 7805. This IC converts the 24VDC coming from the SMPS to 5VDC which prevents the motors from blowing down. However 12V DC motor is used to run the roller. This motor is powered by IC 7812 which will convert the 24VDC coming from the SMPS to 12VDC. An Inductive proximity sensor is used to sense the rotation of the roller. The number of rotations is feedback to the LabVIEW which will convert the number of rotation of the roller into how much meters of cloth has been stretched. The prototype of Stenter Machine is connected to LabVIEW based SCADA via PLC and RS- 232 cable.

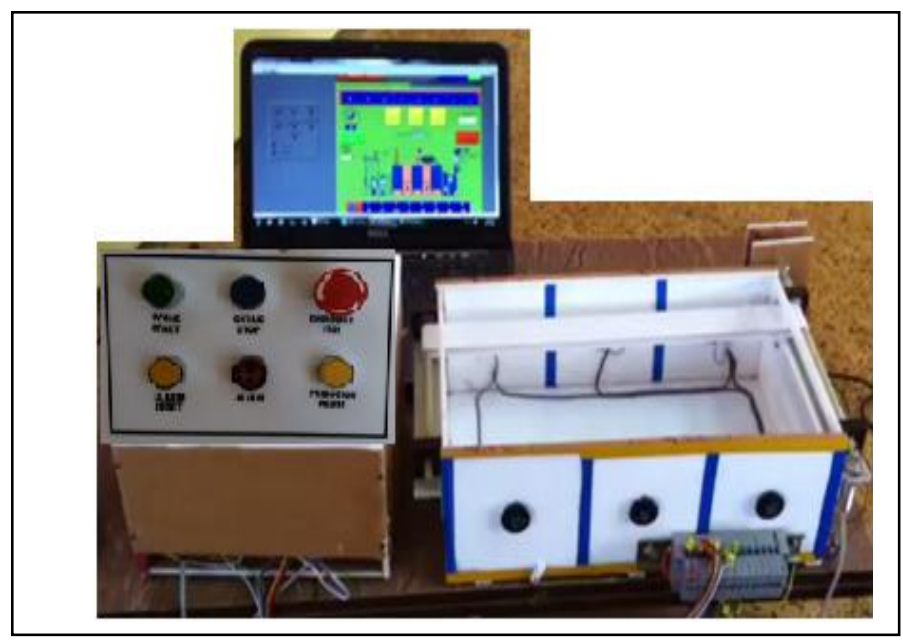

Fig 5 System Hardware Design

\section{DESIGN OF SYSTEM SOFTWARE}

Fig. 6 shows the complete Graphical User Interface designed in LabVIEW 8.5. The first page shows the complete schematic diagram of a Stenter Machine. The indicators will indicate all the conditions of the machine. By turning the knob one can set the values as per how much a cloth has to be stretched. The remaining options will be set according to the set values provided by the operator.

One can even keep the track of the temperature of each chamber of Stenter Machine and the speed of each blower can be set and recorded by this system. In our prototype we have only taken the feedback for roller rotations.

Second page is to view each blower of the Stenter Machine. If the temperature has to be maintained inside the chamber, the blower of that chamber can be switched OFF by pressing just a button on the front panel.

Similarly the third page is to monitor the amount of steam sent into each chamber of the Stenter Machine. If the steam required for specific chamber is different from the other, one can set the speed of the exhaust fan.

Forth page is a GUI for Printing Machine. Printing Machine has a conveyor belt where the cloth is placed. It has different number of trolleys where colors are placed to be printed on the cloth along with different patterns to be printed. The motor run the conveyor belt. An encoder is placed on a roller which would calculate the pulses to move the conveyor belt. At what 
speed a trolley of color should move, at what length of cloth the printing should be done is given by the operator. According to the given speed and length, it calculates the number of pulse to be generated to move the conveyor belt. When all the trolleys of different color and speed come to rest, the roller will run the conveyor belt in forward direction. As the pulse count stops, the conveyor belt will stop and reset to zero and the trolleys will start moving.

Fifth page is a data logging page. Here data can be viewed for minimum one second to maximum one day. It depends on the operator to set the data logging time. The data will be documented as per the difference of time specified by the operator. This data is also stored in an Excel file created by the user. Hence even if the data on LabVIEW is erased, one can have a backup and one can view the data whenever required.

Sixth page is the communication page. The LabVIEW does not calibrate with PLC unless its codes are created in LabVIEW. Hence one cannot change the PLC whenever required. Each PLC has different hex codes which have to be registered into the LabVIEW. The string has to be decided according to the communication protocol. Each PLC has different protocols and addressing. People do not use LabVIEW for this reason. But this problem acts as an advantage and makes the system secured and safe.

The designed GUI is user friendly and informative. It provides all the control actions to be taken at the operator end and provides the operator with information regarding different alarm situations. It also allows data logging and storage for future referral. It communicates with the field controller (PLC) through simple serial communication protocol RS-232 thereby allowing less communication hardware to be used.

\section{IMPLEMENTATION OF DESIGNED SYSTEM}

In this system a Graphical User Interface, LabVIEW is being used as a Supervisory Control and Data Acquisition System (SCADA). It is a measuring software which is used here as a SCADA. It is connected to PLC via RS-232 cable. The PLC acts as an interface between the software and the prototype of a textile machine, which is Stenter Machine.

\subsection{Implementation Algorithm for Manual Operation}

- Initialize SMPS (Switched Mode Power Supply).

- $\quad$ Provide connections between computer, SMPS, PLC and prototype of Stenter Machine.

- Burn the ladder diagram created in WPLSoft 2.20 in PLC.

- Operate manually by pressing the buttons, which is indicated in Fig. 6, on the hardware to check whether the connections are proper.

- Open the door of Stenter Machine to check and verify alarm condition.
- $\quad$ Check all the conditions provided on Fig. 6 and verify the working of the Stenter Machine.

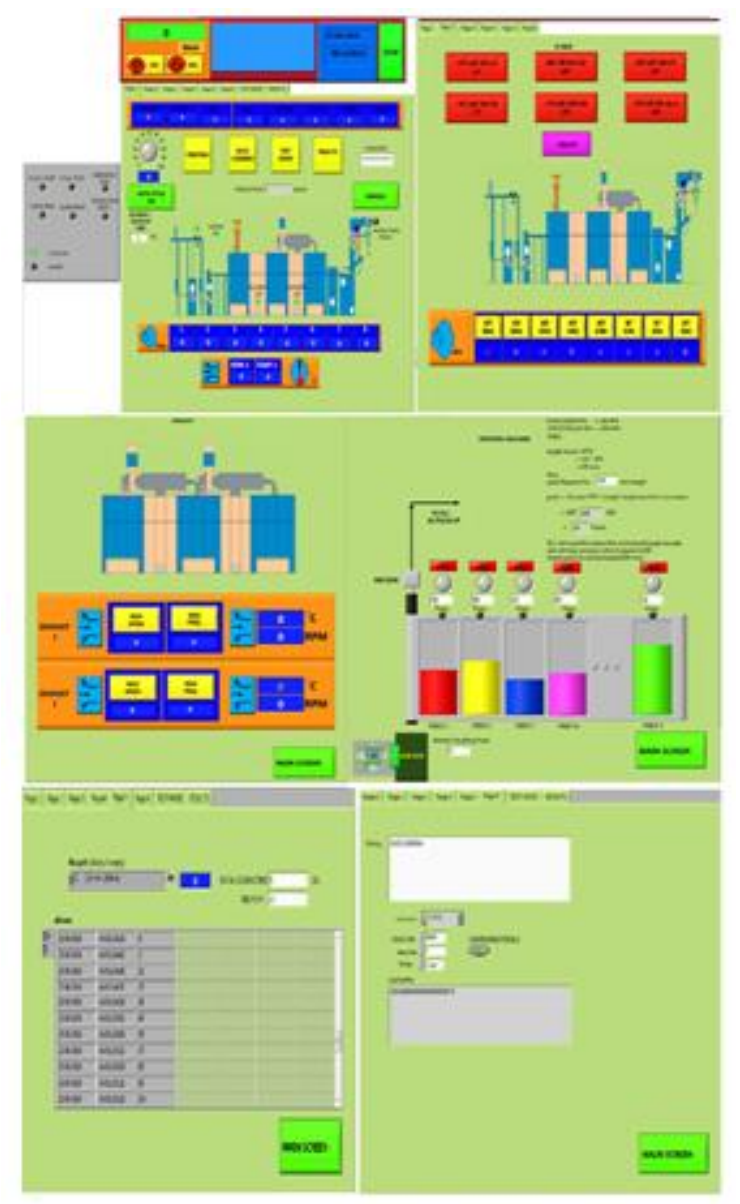

Fig 6 Different Pages of the Designed GUI

\subsection{Implementation Algorithm for Automatic}

\section{Operation}

- $\quad$ Open the SCADA system created in LabVIEW 8.5.

- Initialise the path where the data has to store in spreadsheet format.

- $\quad$ Start the process by pressing Auto Cycle ON. Before pressing it note that the process is on Auto Mode and not on Manual Mode.

- $\quad$ As the cycle starts check the Blower 1 and Blower 2 indicators on figure $61^{\text {st }}$ page of VI. Blower 1 will start as the cycle starts and blower 2 will be $\mathrm{ON}$ after 4 seconds delay.

- Press Printing Machine button and check the machine's working.

- Again go to the first page and press data logging and check the output.

- $\quad$ Again return to the main page and go to the results. 
- In results one can find the relationship between time and production.

Fig. 7 represents a flowchart illustrating operational procedure for the proposed system. The flowchart is the implementation of the algorithm and working of our actual designed system.

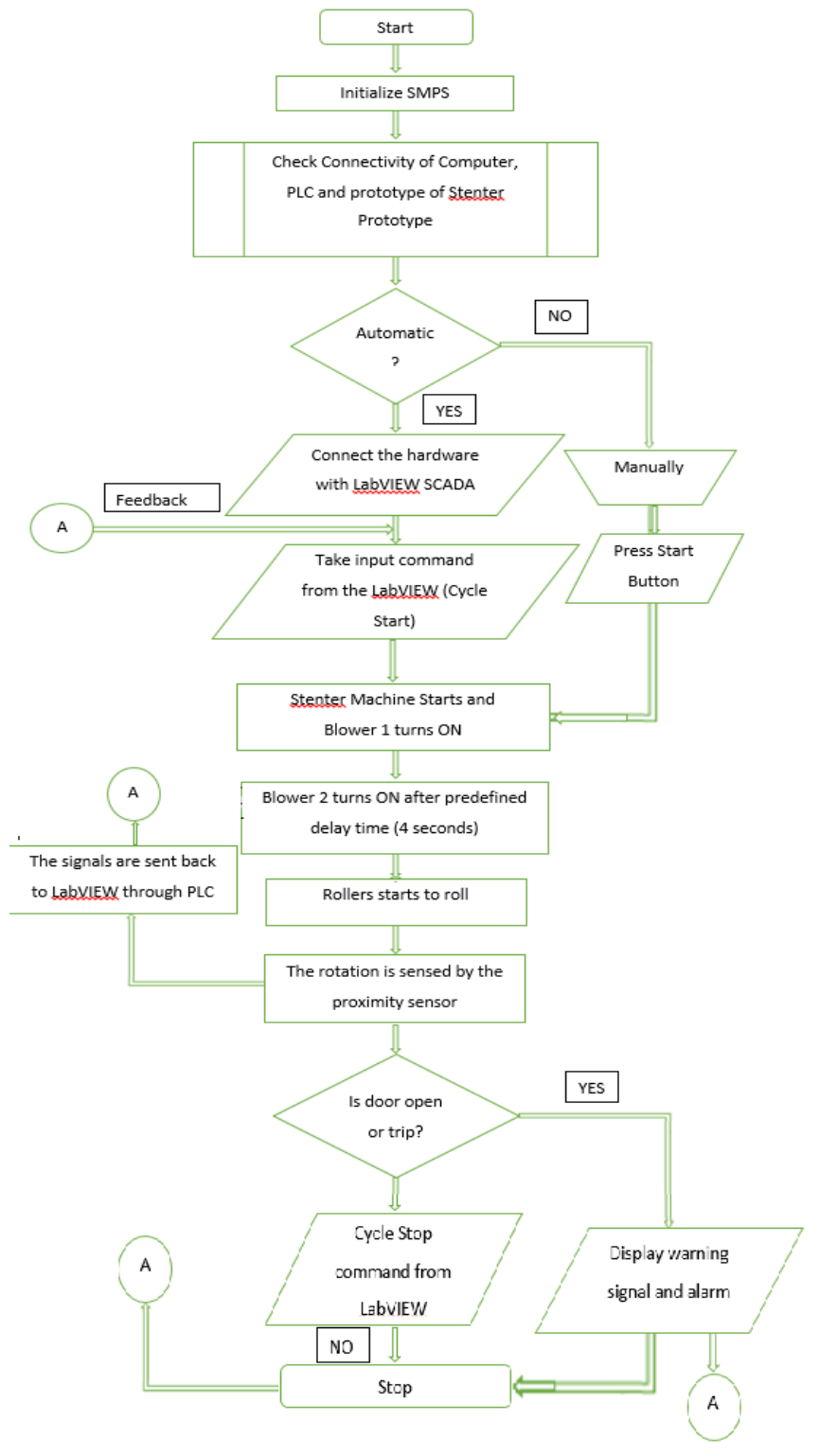

Fig 7 Flowchart

\section{RESULTS AND DISCUSSION}

Fig. 8 shows the relationship between time in seconds and number of rotation of the roller. It is clear that as the time rises the roller at the output of the Stenter machine rotates and therefore the number of rotations per second increases.
Fig. 9 shows the relation between time in seconds and production in meters. The calculation needed to convert the number of rotation into production is given by:

Production $($ meters $)=$ Number of Rotations $* 3.14 *$ Diameter (user defined in $\mathrm{mm}$ ).

The diameter here is user defined as the diameter of output roller where cloth is wound may be different. This point has to consider and accordingly how much meter cloth is needed at the output should be recorded, known and monitored.

Fig. 10, the data $\log$ is created in the Excel file. It helps in recalling the data and keep track on the production even after the machine is stopped.

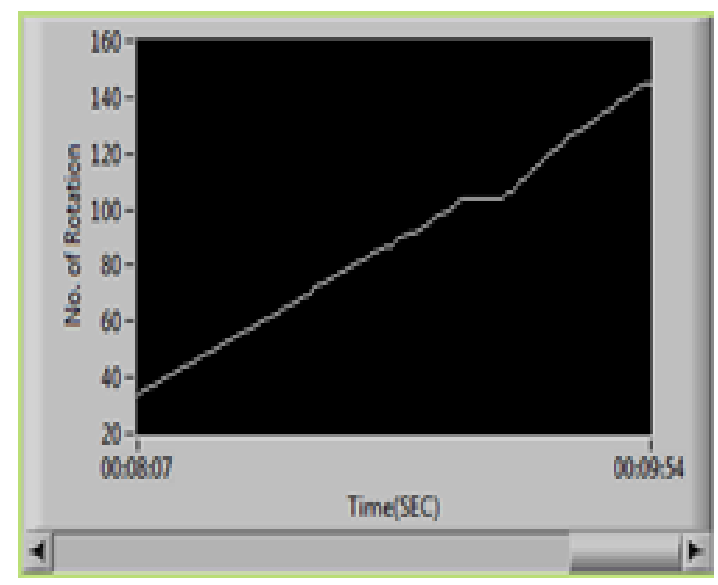

Fig 8 Graphical Representation of Time verses No. of Rotation

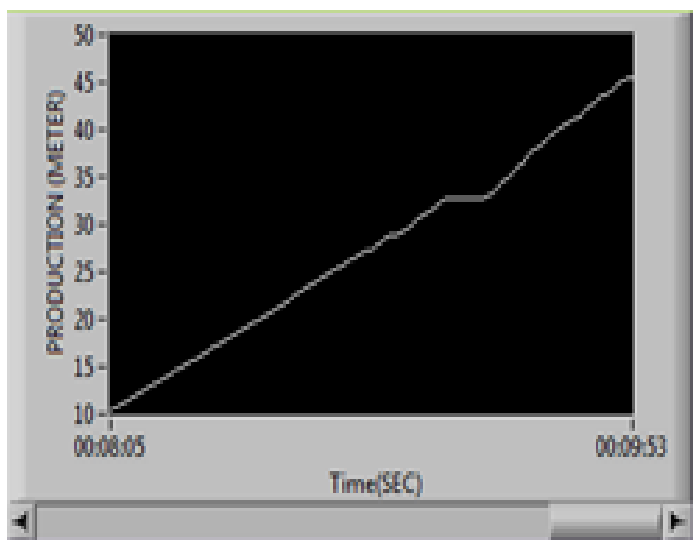

Fig 9 Graphical Representation of Time verses Production (Meter) 


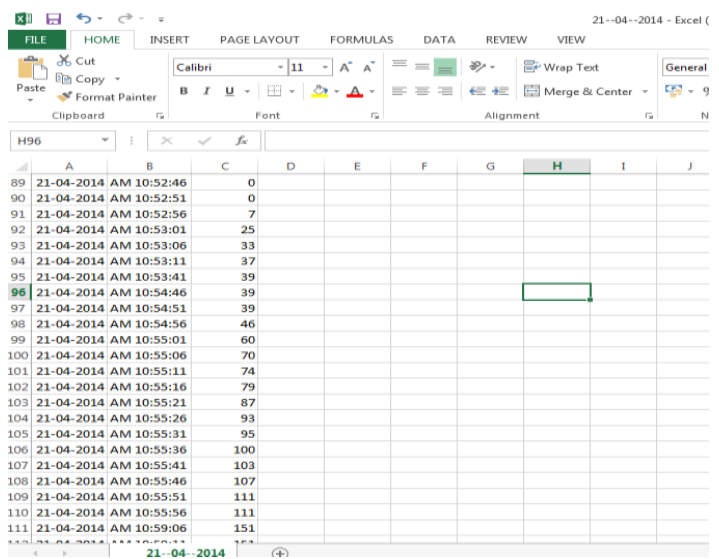

Fig. 10 Data Logging Stored in Excel File

Table 1 indicates different alarm situations occurring due to some emergency situation or safety concerns. The design provides feedback of the emergency situation at both the manual operator panel and the SCADA. It also allows the operator to rest the alarm from the SCADA and manual operator panel. The manual panel is provided for on-site control and over ride in emergency situations.

Table 1: Alarm Situations

\begin{tabular}{|c|c|c|}
\hline Condition & Alarm & $\begin{array}{c}\text { Cycle Start/ } \\
\text { Stop }\end{array}$ \\
\hline $\begin{array}{c}\text { Cycle Start } \\
\text { without any fault }\end{array}$ & OFF & Start \\
\hline $\begin{array}{c}\text { Normal Operating } \\
\text { Condition }\end{array}$ & OFF & Start \\
\hline $\begin{array}{c}\text { Door of Stenter } \\
\text { machine Open }\end{array}$ & ON & Stop \\
\hline $\begin{array}{c}\text { Trip Condition/ } \\
\text { External Fault }\end{array}$ & ON & Stop \\
\hline Emergency Stop & OFF & Stop \\
\hline
\end{tabular}

\section{CONCLUSIONS}

The paper presents the design and implementation of SCADA system using LabVIEW platform for small scale textile industries. The designed SCADA is tested using prototype of Stenter machine. The SCADA provides centralized monitoring and supervisory control of the process. The stenter prototype control is being implemented by a PLC field controller. The PLC acts as RTU and communicates using serial communication protocol with the designed SCADA. The LabVIEW as a programming language offers flexibility in design, making future up gradation less time consuming.

The designed HMI is user friendly which provides all the necessary process information. The process feedback includes the rate of production, operating hours, alarm situation and fail safe condition. The designed system addresses the problem of low cost automation for small scale industries. Many more features such as network control, internet data logging, and internet process control can be easily incorporated in the designed system.

\section{REFERENCES}

[1]. A. Hace, K. Jezernik, Member, IEEE, "Control System for the Waterjet Cutting Machine", IEEE Transaction, Slovenian Ministry of Education, Science and Technology under Grant R55-3355, December 2003.

[2]. Nilima V. Warke, "LabVIEW - Teaching Aid for Process Control", ACEEE Int. J. on Control System and Instrumentation, Vol. 03, No. 02, March 2012.

[3]. V. Lohith Kumar and S.Varsha, "Design and Analysis of Quality Control in Process Industries Using Labview", Int. Journal of Engineering Research and Application Vol. 3, Issue 5, Sep-Oct 2013, pp.469-472.

[4]. Norashikin M. Thamrin and Mohd. Mukhlis Ismail, "Development of Virtual Machine for Programmable Logic Controller (PLC) by Using STEPS Programming Method", IEEE International Conference on System Engineering and Technology (ICSET), 2011

[5]. Yucel Ugurlu, Member, IEEE, Tatsuro Nagano, Y. Ugurlu and Minato-ku, "Project-Based Learning Using LabVIEW and Embedded Hardware", IEEE 2011.

[6]. Naregalkar Akshay, K. Uday Sravanth, Rahul Varanasi and J. Ankitha Reddy, "Real Time Automated Control of Industrial Processes with PLC -LABVIEW Communication", International Journal for Research in Science \& Advanced Technologies, Volume-1, Issue-1, July- August 2012, pp 035038.

[7]. Rajesh Kurnar, Syed Akif Karnal, Furqan M. Khan, "Designing And Implementing SCADA Subsystem for Textile Industry", IEEE, 2004

\section{Authors Biography}

1. Mr. Nirmal Bharatia is a post- graduate (Electronics) student at Mukesh Patel School of Management and Engineering, SVKM's NMIMS University. He completed B.E. (E.E.) from VNSGU. He has a work experience in core electrical field for past 3 years.

2. Mr. N.D.Thakur is working as assistant professor in Electronics \& Telecommunication Engg. Department of MPSTME, NMIMS, Mumbai. He has completed B.E. (I.E.), M.Tech. (Electronics) and M.B.A. (H.R.) and currently pursuing P.H.D. in Electronics. He has more than 10 years of academic experience and published several papers in national and international journals. His areas of interest are automation, process control and biomedical signal processing. 